\title{
Classification of Object Tracking Techniques in Wireless Sensor Networks
}

\author{
Mohsin Fayyaz \\ Department of Electrical Engineering, COMSATS Institute of Information Technology, Abbottabad, Pakistan \\ E-mail: mohsin1900@gmail.com \\ Received February 19, 2011; revised March 9, 2011; accepted March 15, 2011
}

\begin{abstract}
Object tracking is one of the killer applications for wireless sensor networks (WSN) in which the network of wireless sensors is assigned the task of tracking a particular object. The network employs the object tracking techniques to continuously report the position of the object in terms of Cartesian coordinates to a sink node or to a central base station. A family tree of object tracking techniques has been prepared.In this paper we have summarized the object tracking techniques available so far in wireless sensor networks.
\end{abstract}

Keywords: Object Tracking in WSN, Classification of Object Tracking Techniques in WSN

\section{Introduction}

The techniques are mainly classified based on (see Figure 1)

- Network architecture used

- Algorithm or technique used

- Type of sensors used

- Number of targets to be tracked.

- Technology used for implementation.

Each of these categories has been described carefully in section 2, 3, 4, 5 and 6 respectively.

\section{Network Architecture}

The following are the main types of network architectures that can be used for object tracking in sensor networks as shown in Figure 2.

- Cluster based architecture.

- Decentralized architecture.

- Tree based architecture.

\subsection{Cluster Based Architecture}

In cluster based architecture there are several sensor nodes and for a certain group of nodes, they are assigned a cluster leader or cluster head. The ordinary nodes just sense the reading and send them to the cluster thereby shifting the burden from them to the cluster head. The cluster head is normally a high energy and high resource node. The introduction of cluster heads can reduce suffi- cient cost of network as one can deploy low cost low energy sensor nodes [1].

The cluster based architecture of wireless sensor networks can also be classified as

- Static clustering

- Dynamic clustering

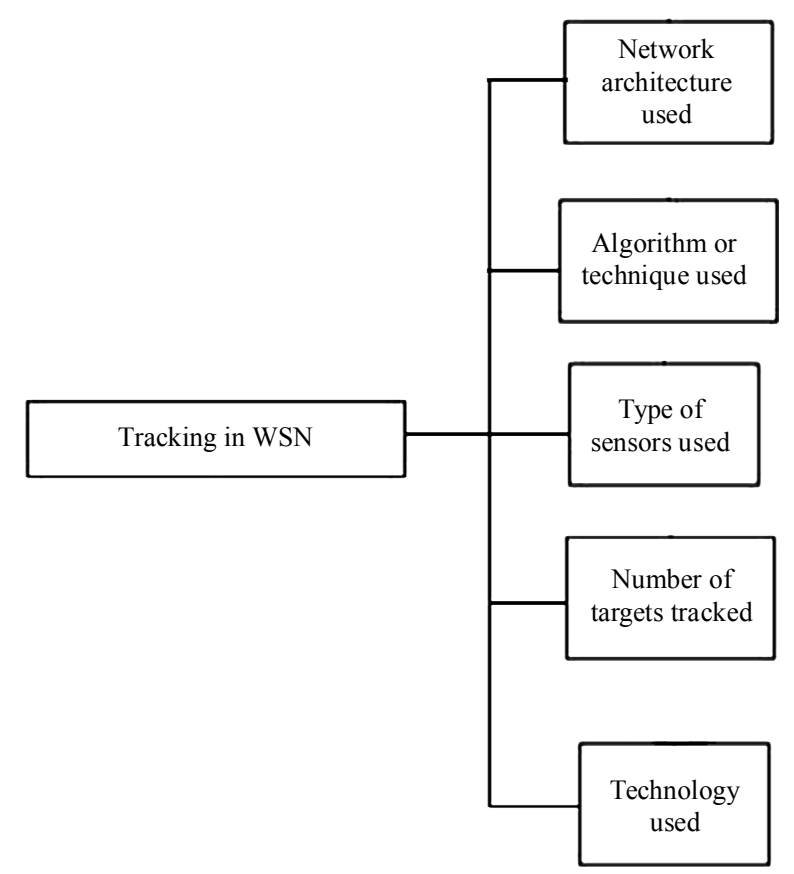

Figure 1. Basic classification of tracking techniques in WSN. 


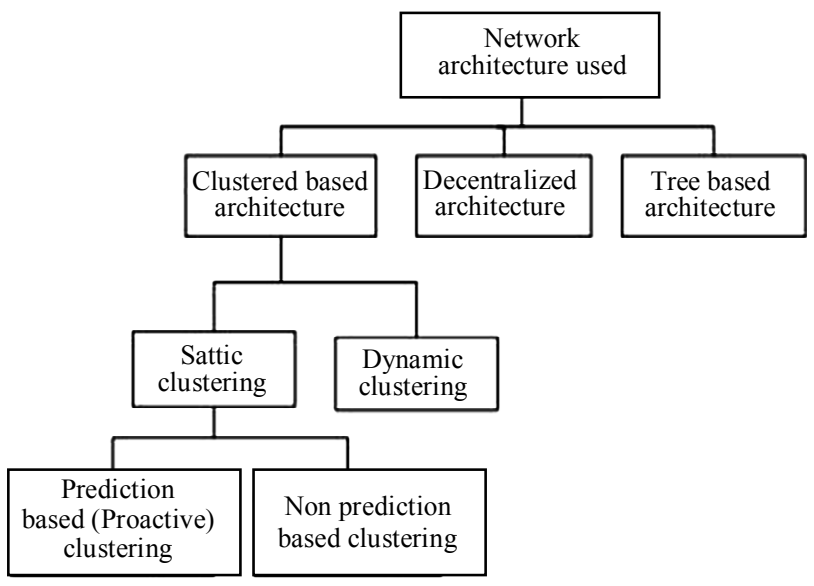

Figure 2. Further classification of techniques on the basis of network architecture used.

\subsubsection{Static Clustering}

In static clustering the cluster heads are assigned to the respective sensor nodes at the time of formation of network and they can not be changed. This means that throughout the working of wireless sensor network the nodes remain attach to the same cluster head as they were pre assigned [2].

\subsubsection{Dynamic Clustering}

Again the dynamic clustering scheme can be of two types

- Prediction based or Proactive clustering.

- Non prediction based.

\subsubsection{Prediction Based or Proactive Clustering}

This scheme is mostly employed in a network of sleep sensors, where most of the sensors stay in the sleep mode. In prediction based clustering when a target moves from the region of one cluster head to the other, the current cluster head has to make an estimation or prediction about where the target is moving and correspondingly wakeup a cluster head where the target is moving $[1,3]$.

\subsubsection{Non Prediction Based Clustering}

This is similar to the scheme described under the heading of dynamic clustering. This scheme is used in a network of non sleep sensors. Here the energy saving is not an issue instead the proper selection and the life time of cluster head is an issue. So based in some criteria based on the application environment a cluster head selection algorithm is run on each individual node, and the nodes collaboratively select the cluster head.

\subsection{Decentralized Architecture}

In the decentralized architecture, there is no cluster head type of central entity in the network and in this case all the network nodes are at the same level in terms of work responsibility. So the information regarding the target localization travels through the network to a central base station that is not the part of wireless sensor network. That base station can be a computer or some other computation entity. It runs an algorithm through which it can estimate the current location of the target [4].

\subsection{Tree Based Architecture}

In this case a tree structure is maintained across the network. The tree is rooted at the node that is closest to the target. Thus as the target moves some nodes get added to the tree and some get deleted. This scheme reduces the overhead in terms of energy and information flow, as the information flows from the root to the end or periphery of the network through a particular route, as the information flows is controlled so energy consumption automatically gets controlled [5-9].

\section{Algorithm or Technique}

According to algorithm or technique used the tracking techniques can be classified as (see Figure 3)

- For the network of sleepy sensors.

- Target reporting.

- Target chasing.

For the network of sleepy sensors there is a further classification based on whether prediction heuristics are used or sensor scheduling is used.

\subsection{For the Network of Sleep Sensors}

As all the sensors are in sleep mode. Thus there needs to be a mechanism through which the sensors can be woken up when the target approaches in the region of sleepy sensors. To wake up either of the methods can be used:

- Prediction heuristics.

- Sensor scheduling.

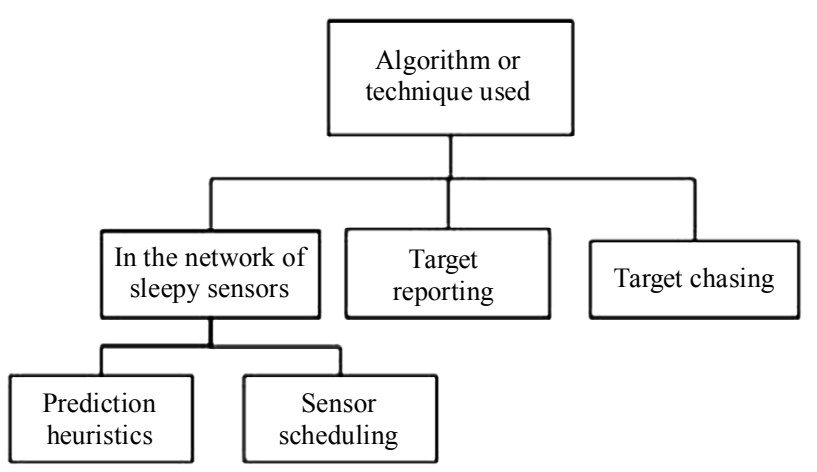

Figure 3. Classification of tracking techniques in WSN according to algorithm or technique used. 


\subsubsection{Prediction Heuristics}

In this case most of sensors stay in sleep mode. The current node predicts the future movement and location of the target and correspondingly wakes up the nodes in the region where the target is moving. The critical performance parameters for prediction based reporting are the miss rate and energy consumption. The network or the regions where the current wireless sensor nodes were active.

The heuristics reflect the prediction model that can be used for the prediction based object tracking in WSN.

The prediction heuristics that can be used can either of

Heuristic INSTANE

In this heuristic it is assumed that the target's future speed and direction will be the same as it is currently having.

\section{Heuristic AVERAGE}

Here it is assumed that the future direction and movement of the target will be equal to the average of previous direction and movement.

\section{Heuristic EXP_AVG}

Here also the average of past readings is carried out except it is exponential weighted average, which means that more weight is assigned to near future reading than the far future readings [1].

\subsubsection{Sensor Scheduling}

Instead of incorporating prediction based scheme sensors can be scheduled for their wakeup and sleep time.

In this scheme it is to be determined that which sensors stay in awake over the time in order to have an appropriate trade off between the tracking performance and the overall sensor usage. The objective is to minimize the estimation errors while still reducing the sensor usage over a period of time [10].

\subsection{Target Reporting}

In target reporting only the information target location in terms of Cartesian coordinates is sent to a sink node or some central entity. Thus the data continuously travels through the network. Thus the main task here is to device efficient routing and target location calculation tecniques in order to minimize the overall energy consumption by the network and minimizing the tracking error [11].

\subsection{Target Chasing}

In target node the sink node has to physically follow the target. Thus the sink node has to continuously consult the neighboring nodes and the information of target has to be disseminated in the network for sink to follow the target [10].

\section{Type of Sensors}

The tracking techniques are widely different depending on the type of sensors used: (see Figure 4)

- Ordinary sensors.

- Binary sensors.

\subsection{Ordinary Sensors}

The ordinary sensor network consists of the type of sensor nodes that operate on original values of signals. Thus the distance, speed and direction of target have to be calculated on the basis of signal strength measured by the sensor nodes.

\subsection{Binary Sensors}

The binary sensors work only on two binary values. They can just detect the presence or absence of the target in their sensing range by signaling either by 1 or 0 . Thus the tracking mechanism in this case is more complicated than ordinary sensor networks $[4,12]$.

\section{Number of Targets Used}

The tracking technique can be either for single target tracking or multiple target tracking. (see Figure 5)

\subsection{Single Target Tracking}

Tracking in single target is relatively simple. Less data is produced that results in a low traffic in the network. Less traffic is easier to handle and the routing mechanism is not complex.

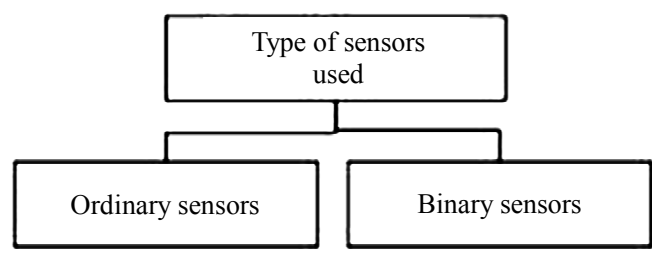

Figure 4. Classification of tracking techniques on the basis of types of sensors.

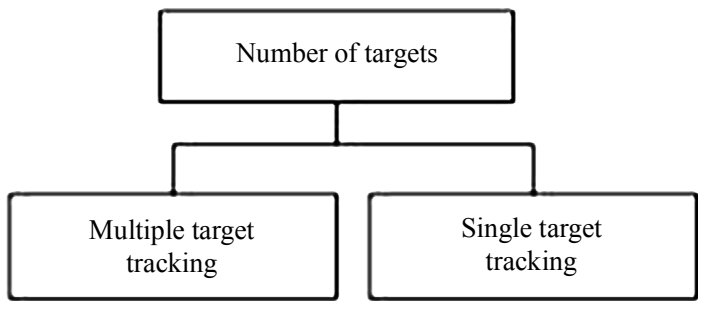

Figure 5. Classification on the basis of number of targets tracked. 


\subsection{Multiple Target Tracking}

In this case the location of multiple targets has to be tracked simultaneously. Increasing the number of targets to be tracked increases the network traffic and thus more complex routing schemes and energy minimization tecniques have to be incorporated to compensate for the network performance [13].

\section{Technology Used for Implementation of Tracking in Wireless Sensor Networks}

Various technologies can be used for implementation of tracking techniques in wireless sensor networks including Zigbee, Bluetooth etc. Mostly the technology which uses omni directional antennas can be used for the implementation of tracking in wireless sensor networks. This is because the presence of target has to felt, what ever the direction of motion of target is. The omni directional antennas solve the purpose of tracking.

\section{Conclusion and Results}

- A wide range of technologies, network architectures and types of sensors are available for tracking in wireless sensor networks.

- There is a performance tradeoff in energy, tracking error and other performance parameters.

- To design a wireless sensor network for object tracking or to do research in proposing new techniques the classification of techniques has to be kept in to mind with their relative trade offs to achieve affective results.

\section{References}

[1] Y. Q. Xu, J. L. Winter and W.-C. Lee. "Prediction Based Strategies for Energy Saving in Object Tracking Sensor Networks," Proceedings of IEEE International Conference on Mobile Data Management, Berkeley, 19-24 January 2004, pp. 346-357.

[2] H. Yang and B. Sikdar. "A Protocol for Tracking Mobile Targets Using Sensor Networks," Proceedings of IEEE Workshop on Sensor Network Protocols and Applications Anchogare, May 2003, pp. 71-81. doi:10.1109/SNPA.2003.1203358

[3] E. Olule, G. J. Wang, M. Y. Guo and M. X. Dong, "RARE: An Energy-Efficient Target Tracking Protocol for Wireless Sensor Networks," Prceedings of IEEE International Conference on Parallel Processing Workshops, Xian, 10-14 September 2007, pp. 76-82.

[4] J. Aslam, Z. Butler et. al. "Tracking a Moving Object with a Binary Sensor Network," Proceedings of the 1st International Conference on Embedded Networked Sensor Systems, Los Angeles, 5-7 November 2003, pp. 150-171. doi:10.1145/958491.958509

[5] C.-Y. Lin, W. -S. Peng and Y.-C. Tseng, "Efficient In Network Moving Object Tracking in Wireless Sensor Networks," IEEE Transactions on Mobile Computing, Vol. 5, No. 8, August 2006, pp. 1044-1056.

[6] H. T. Kung and D. Vlah. "Efficient Location Tracking Using Sensor Networks," Proceedings of IEEE Wireless Communications and Networking Conference, New Orleans, Vol. 3, March 2003, pp. 1954-1961.

[7] W. S. Zhang and G. H. Cao. "DCTC: Dynamic Convoy Tree-Based Collaboration for Target Tracking in Sensor Networks," IEEE Transactions on Wireless Communications, Vol. 3, No. 5, September 2004, pp. 1689-1701.

[8] C.-Y. Lin and Y.-C. Tseng. "Structures for In-Network Moving Object Tracking in Wireless Sensor Networks," Proceedings of the 1st International Conference on Broadband Networks, San Jose, 25-29 October 2004, pp. 718-727.

[9] W. S Zhang, G. H. Cao. "MobiHoc Poster: Optimizing Tree Reconfiguration to Track Mobile Targets in Sensor Networks," Mobile Computing and Communications Review, Vol. 7, No. 3, July 2003, pp. 39-40.

[10] Mohsin Fayyaz. "Object Tracking in Wireless Sensor Networkls," MSc Project Report, Department of Electronic Engineering, Queen Mary University of London, London, 2008.

[11] T. He, S. Krishnamurthy, et. al. "Energy-Efficient Surveillance System Using Wireless Sensor Networks," Proceedings of the 2nd ACM International Conference on Mobile Systems, Applications and Services, Boston, 6-9 June 2004, pp. 270-283. 1-58113-793-1/04/0006 doi:10.1145/990064.990096

[12] N. Shrivastava, R. Mudumbai, et. al. "Target Tracking with Binary Proximity Sensors: Fundamental Limits, Minimal Descriptions and Algorithms," Proceedings of the 4th ACM International Conference on Embedded Network Sensor Systems, Boulder, 1-3 November 2006, pp. 251-264.

[13] S. Oh, L. Schenato, et. al. "A Hierarchical Multiple-Target Tracking Algorithm for Sensor Networks," Proceedings of IEEE International Conference on Robotics and Automation, Barcelona, 18-22 April 2005, pp. 2197-2202. 